\title{
Solvent effects on the harvesting of stratum corneum from hairless mouse skin through adhesive tape stripping in vitro
}

\author{
Jui-Chen Tsai ${ }^{1}$, Markus J. Cappel ${ }^{2}$, Norman D. Weiner ${ }^{1}$, Gordon L. Flynn ${ }^{1}$ \\ and James Ferry ${ }^{3}$ \\ ${ }^{\prime}$ College of Pharmacy, University of Michigan, Ann Arbor, MI 48109 (U.S.A.) ${ }^{2}$ Institut für Pharmazeutische Technologie, \\ J.W. Goethe Universität (Germany) and ${ }^{3}$ The Upjohn Company, Kalamazoo, MI (U.S.A.)
}

(Received 19 April 1990)

(Modified version received 23 August 1990)

(Accepted 5 September 1990)

Key words: Solvent effect; Tape stripping; Stratum corneum; Topical application

\begin{abstract}
Summary
$125 \mu \mathrm{l}$ of a propylene glycol/ethanol/water vehicle were applied for various lengths of time to $1.77 \mathrm{~cm}^{2}$ area of excised hairless mouse skin sections held in in vitro diffusion cells. After removal from the cell, each skin section was stripped repeatedly with a non-hygroscopic polypropylene tape. The amount of tissue removed in each strip was determined after allowing the volatile solvents to evaporate. Weights were corrected for residual propylene glycol and water, the amounts of which were determined radioisotopically. More tissue was harvested in the first and second strips from skin conditioned with the vehicle for more than $12 \mathrm{~h}$. The effect of vehicle treatment on stripping properties precludes one from determining drug and vehicle concentration gradients in the stratum corneum at different treatment times by direct comparison of corresponding strips. While it does not appear that a penetrant's depusition as a function of time can be followed easily and directly by stripping and then quantifying the drug (or solvent) in the respective layers, the stripping technique may still be useful in separating stratum corneum (or epidermis) from dermis.
\end{abstract}

\section{Introduction}

It has long been known that stratum corneum, the outermost skin layer, can be removed by repetitive application of an appropriate adhesive tape to a given skin surface area (Pinkus, 1951). In principle, if the strippings are uniform, the drug amount can be measured in each single tape strip to obtain a concentration profile within the horny layer. The stripping technique can be used to gain some insight into drug concentration no matter

Correspondence: N.D. Weiner, College of Pharmacy, University of Michigan, Ann Arbor, MI 48109, U.S.A. whether the application was done in vitro or in vivo (Schalla and Schaefer, 1985). It has been reported, however, that the amount of tissue removed by each consecutive tape stripping decreases (Tregear and Dirhuber, 1962; Zesch et al., 1973). Thus, to obtain the best estimate of the prevailing concentration gradient, the amount of drug measured in each tape strip should be normalized with respect to the amount of tissue removed in each individual strip. Moreover, to make the best comparisons of formulations, the amounts of tissue lifted off the skin per strip should be near the same and formulation independent.

Recently, we have shown the effect of vehicle composition on the percutaneous delivery of 
minoxidil through hairless mouse skin using in vitro diffusion studies (Chiang et al., 1989). In an effort to extend these studies, we attempted to use the stripping technique to determine the concentration profiles of minoxidil in the stratum corneum upon application of minoxidil solution (Rogaine $^{\otimes}$ Solution, The Upjohn Company, Kalamazoo, MI) to the skin for various lengths of time. Early results clearly indicated that when the drug solution was applied to the skin surface for long period of time, the amount of tissue adhering to a tape strip appeared to change, making strip by strip comparisons questionable. The purpose of this paper is to determine what effect the vehicle may have on skin stripping properties as a function of time. A vehicle composition similar to that

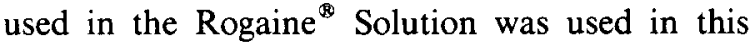
study.

\section{Materials and Methods}

\section{Preparation of the vehicle}

The vehicle consisted of $20 \%$ propylene glycol, $60 \%$ ethanol and $20 \%$ water by volume. $\left[{ }^{14} \mathrm{C}\right]$ Propylene glycol and $\left[{ }^{3} \mathrm{H}\right]$ water were incorporated into the vehicle to account for the presence of solvent residues when determining the amount of skin harvested in each tape stripping. The resulting vehicle contained approx. $1.5 \mu \mathrm{Ci}\left[{ }^{3} \mathrm{H}\right]$ water and $1.0 \mu \mathrm{Ci}\left[{ }^{14} \mathrm{C}\right.$ propylene glycol per $\mathrm{ml}$. $\left[{ }^{14} \mathrm{C}\right]$ Propylene glycol and $\left[{ }^{3} \mathrm{H}\right]$ water were purchased from ICN Radiochemicals, Irvine, CA.

\section{Preparation of the skin membrane}

Freshly prepared full-thickness hairless mouse skin obtained from 60-90 days old hairless mice (strain Skh-hr-1, Central Animal Facility, Temple University, Philadelphia, PA) was used in the solvent treatment experiments. The animals were sacrificed by cervical dislocation and abdominal and dorsal skin sections were excised. Adhering subcutaneous fat on the dermal side was carefully removed from the skin's underside.

\section{Preparation of the adhesive tape}

Although cellophane tape (Scotch ${ }^{\mathrm{TM}}$ brand No. $810,3 \mathrm{M}$ Co., St. Paul, MN) is commonly used in

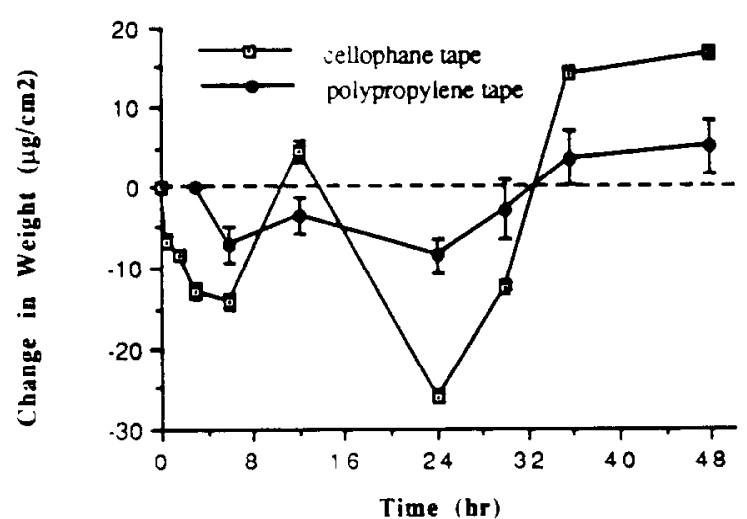

Fig. 1. Comparison of weights of cellophane tape and polypropylene tape as a function of time. All weight shifts are normalized to a $1 \mathrm{~cm}^{2}$ area and are referenced to the initial weight. Fach data point indicates the mean value \pm the standard error of the weights $(n=4-6)$.

stripping horny layer tissue from the skin, rapidly fluctuating weight as a result of its hygroscopicity precludes valid measurement of the tissue mass adhering to the tape (Han et al., 1989-1990). A polypropylene tape $\left(\mathrm{Scotch}^{\mathrm{TM}}\right.$ brand No. $845,3 \mathrm{M}$ Co.), which is reputedly less hygroscopic, was selected as the stripping tape because of similar adhesiveness to the ordinary cellophane tape and because it readily offered measurement of the tissue mass through weighing. The relatively stable weights of the polypropylene tape sections kept in an open atmosphere reveals that it is suitable for such purposes (Fig. 1). The stripping properties of these two tapes were similar as determined by transepidermal water loss (TEWL) of stripped sites on live mice and distribution profiles of minoxidil in 10 consecutive strips (Tsai et al., 1990).

Tapes were prepared as shown in Fig. 2. First, an oversized piece of Parafilm ${ }^{\star}$ was cut and placed with its clean side facing up. A piece of slightly oversized aluminium foil punched with holes of 19 $\mathrm{mm}$ diameter was placed on top of the film. The polypropylene tape, having a 1.5 inch width was affixed to the Parafilm ${ }^{\circledR}$ and the aluminium foil. The tape, with its accompanying foil and film, was then cut into a circular shape with a punch of 22 $\mathrm{mm}$ in diameter so that a foil edge of uniform thickness formed a ring around the periphery of 


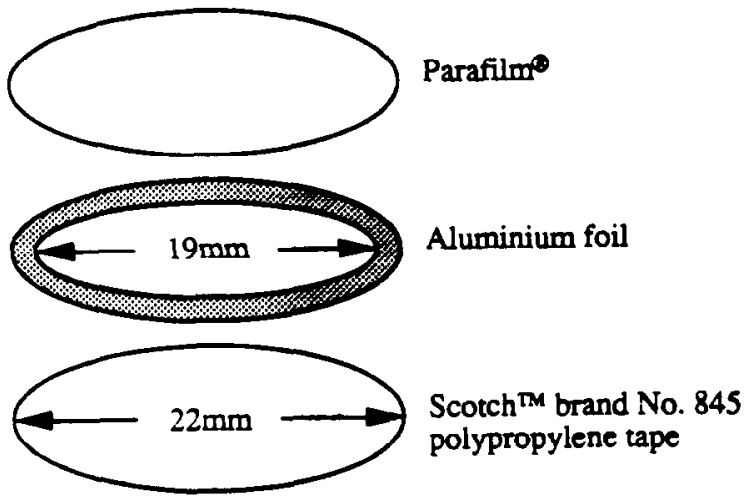

Fig. 2. Diagrammatic illustration of the polypropylene tape preparation.

the tape stripping unit. Thus, each tape used for stripping had an adhesive area of $19 \mathrm{~mm}$ diameter and a $1.5 \mathrm{~mm}$ width of foil around its edges. The latter was used solely for the purpose of easy handling of the individual strip. At least $2 \mathrm{~h}$ prior to stripping of the skin's surface, the Parafilm was removed from the tape. The tape was then placed in an empty scintillation vial in a tray and covered to avoid dust contamination. $2 \mathrm{~h}$ proved a sufficient time for the adhesive of the stripping units to equilibrate with the laboratory atmosphere.

Determination of the amount of tissue removed in each individual tape stripping from hairless mouse skin

The purpose of the study was to assess if vehicle conditioning time of a skin section in the diffusion cell affected the stripping attributes of the skin. Stripping was performed on untreated skin sections and skin sections treated with vehicle. The contact time of the vehicle varied from close to zero to $32 \mathrm{~h}$. Franz diffusion cells (Crown Glass Co., Somerville, NJ), with a diffusional area of $1.77 \mathrm{~cm}^{2}$, were used. The receiver medium consisted of freshly prepared isotonic $\mathrm{pH} 7.4$ phosphate buffer which was stirred at $600 \mathrm{rpm}$ throughout the course of the experiments. A uniform temperature of the receiver medium was maintained at $37^{\circ} \mathrm{C}$ by circulating water through the cell's jacket. Each skin section was clamped in a diffusion cell with its dermal side in contact with the receiver medium. $125 \mu 1$ of vehicle were applied, an amount sufficient to cover $15 \mathrm{~mm}$ diameter of exposed skin. At suitable time intervals, skin was removed from the diffusion cells, its surface rinsed with $2 \mathrm{ml}$ of $70 \%$ alcohol and allowed to air dry for a few minutes. Then, one after another, the prepared polypropylene tape sections were applied over the conditioned skin area, pressed tightly to the skin with the handle of a scalpel, and then pulled away with forceps. Each skin section was stripped 10 times in succession. The reason for choosing a larger diameter $(19 \mathrm{~mm})$ of the adhesive tapes than the initial skin area was to accommodate for swelling and stretching of the skin which occurred over long periods of time. The weight of each piece of tape was measured before stripping. All the weight measurements were performed with a Mettler UM3 microbalance (Mettler Instrument Co., Hightstown, NJ). After stripping, the tape strips were allowed to equilibrate at ambient conditions for 4-5 days to allow evaporation of propylene glycol and water so that stable weights could be obtained. Care was taken to keep the surface clean during this time period. Several blank tapes were also kept under the same conditions to serve as background corrections. After the weights of the strips were measured, each strip was digested in a tissue oxidizer (Model 306 Packard Oxidizer, Packard Instrument Co., Downers Grove, IL), forming water and carbon dioxide. Tritiated water was condensed and collected in the tritium sample vial and Monophase S (Packard Instrument Co.,) was added as scintillator. Radioactive carbon dioxide was then taken up by a carbon dioxide absorbent, Carbosorb (Packard Instrument Co.) and flushed into the $\mathrm{C}-14$ sample vial using the $\mathrm{C}-14$ scintillator, Permafluor V (Packard Instrument Co.). The samples were then counted in a liquid scintillation counter (Beckman LS 9000, Beckman Instruments, Fullerton, CA). The amount of propylene glycol and water residue in each strip was thus determined and the weight of tissue removed in each strip was then calculated by subtracting the amount of propylene glycol and water from the weight increase in the strip. 


\section{Results}

The weights of tissue and vehicle residues in each tape strip after vehicle treatment for various lengths of time are shown in Fig. 3. After evaporation for 4-5 days, virtually all the water in the tissue evaporates but some propylene glycol residue remains. Since ethanol is even more volatile than water, there seemed little reason to assess its residual concentration. There is essentially no difference in the weights of strips taken from untreated skin and skin exposed to the solvent medium for a brief moment only (zero time). For the untreated and zero time skin, the amounts of tissue removed from the third or fourth to the tenth strip was approximately constant and less than that removed from the first few strips. This result agrees with the finding of Zesch et al. (1973) who stripped human skin following application of an ointment for $100 \mathrm{~min}$.

Data plotted in Fig. 3 suggests that the stripping characteristics of the skin are changing with vehicle treatment time. The total amount of tissue removed in 10 strips when determined for each time treatment appeared to increase with time. Fig. 4 shows this trend and when the data was analyzed by simple linear regression, the best fit line has a correlation coefficient much less than unity $\left(r^{2}=0.38\right)$, suggesting the trend may not be linear. However, the slope of the line is statistically highly different from zero $(p<0.001)$, strongly suggesting that total tissue removed actually increases with time. The increase was more obvious from 0 to $1 \mathrm{~h}$ and after $16 \mathrm{~h}$. For the $32 \mathrm{~h}$ treatment, the total amount of tissue harvested doubled as compared to zero time treatment.

\section{Discussion}

Stripping with adhesive tapes was claimed long ago to remove a single sheet of horny layer from skin's surface (Pinkus, 1951). The stripping technique was adopted by Schaefer et al. (1978) to obtain drug distribution profiles in the stratum corneum. If such stripping removes a relatively complete, single sheet of horny cells at a time, drug concentration gradients in the stratum corneum could be determined by measuring the amount of drug in each strip. Such a concentration profile would be useful in interpreting reservoir effects and transport through the skin. However, it can be seen from results of this study that uniform stripping is not achieved. As claimed by Pinkus (1951), the sheets of cells adhering to the strips were not complete (with an estimated one-half to two-thirds of skin surface being stripped) and the amount of horny layer material appears to decrease with increasing depth (Tregear and Dirhuber, 1962; Zesch et al., 1973).

By applying a simple propylene glycol/ ethanol/water vehicle system as a thin film, we demonstrate here that the vehicles in the topical formulations change the skin's stripping properties in that both the stripping profile and total harvest are conditioning time sensitive. Since an increasing amount of tissue is obtained with increasing treatment time of the skin with formulation, there is an implication that more layers of horny cells are being stripped in the earlier strippings for skin undergoing longer treatment times. Thus, the harvest for a given strip will be dependent on the prior strips. More tissue would be likely to be removed in the total of ten strips if some earlier strips remove more layers or if stripping after long formulation incubation leads to lifting of layers not usually recovered, i.e., viable tissue. This result agrees with the suggestion by Weigand and Gaylor (1973) that prior hydration of stratum corneum facilitates its removal by cellophane tape stripping. Removal of the hydrated stratum corneum can be accomplished with about one-third the number of strips ordinarily required. Whatever is occurring here, the important point is that we cannot compare individual strips with corresponding strips for different treatment times since there is no comparable physical meaning to each strip. On this basis it appears that the stripping method may not be suitable to determine and compare skin strata profiles as a function of application time when vehicle effects on the skin are significant. However, its value to remove stratum corneum only, which in turn enables the separation of stratum corneum and dermis, cannot be overlooked. 

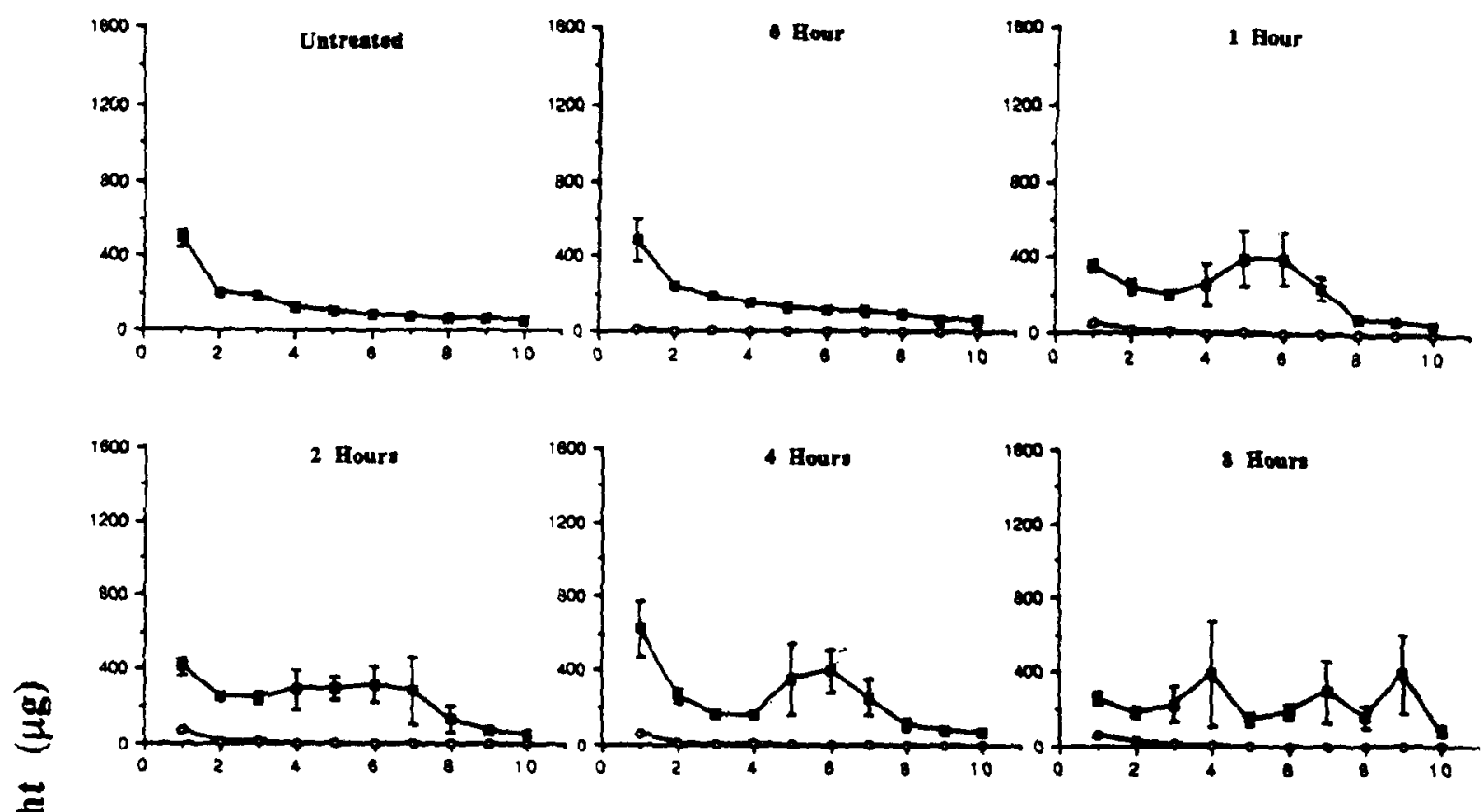

$\frac{2}{3}$
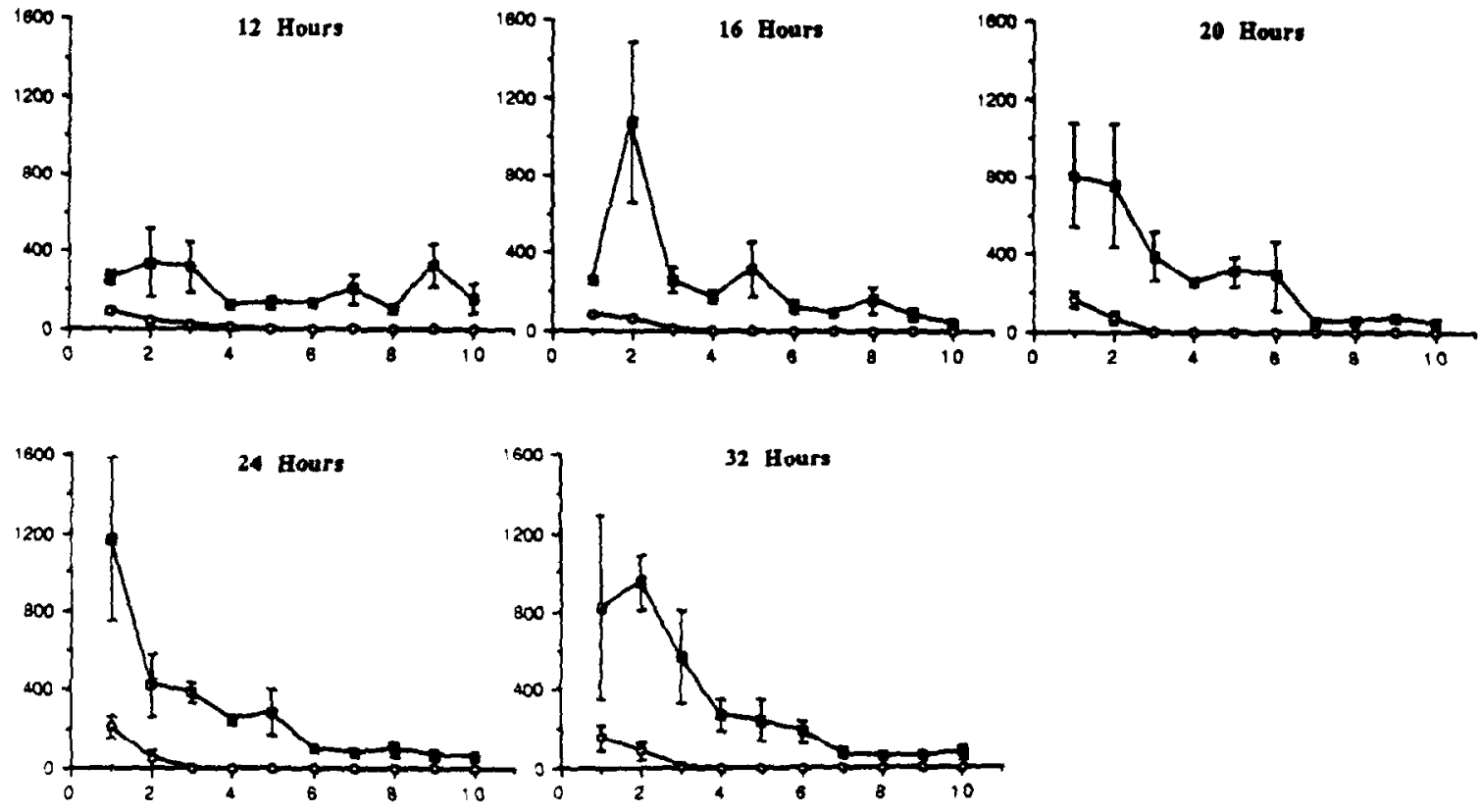

No. of Strippings

Fig. 3. Amount of tissue removed by successive strippings of the same area of the hairless mouse skin treated with $125 \mu$ of propylene glycol/ethanol/water $(20: 60: 20 ; \mathrm{v} / \mathrm{v})$ for different time periods. The weights of residual propylene glycol are also included. Weights of residual water are not shown since no significant amounts $(<3 \mu \mathrm{g})$ were detected. Each data point represents a mean of three measurements \pm the standard error. (ㅁ) skin, (O) propylene glycol. 


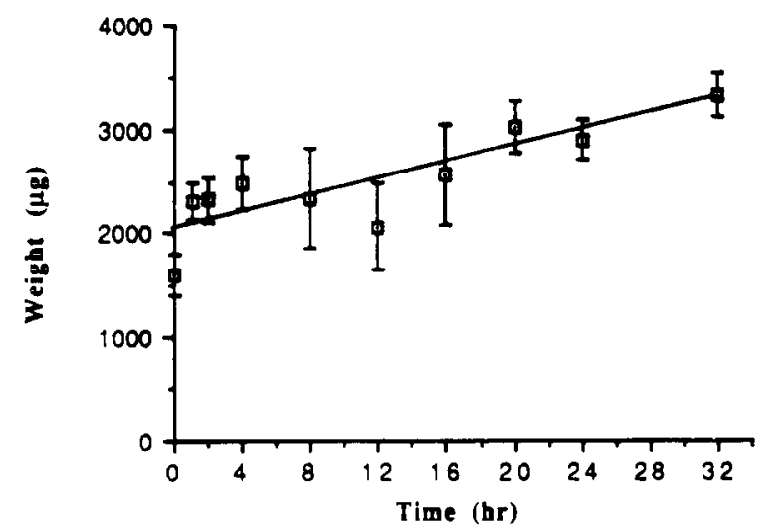

Fig. 4. Total amount of tissue removed in ten strips from hairless mouse skin treated with $125 \mu$ l of propylene glycol/ ethanol/water $(20: 60: 20 ; \mathrm{v} / \mathrm{v})$ for different periods of time. Each data point represents a mean of three data sets \pm the standard error. The best line was drawn through the data by simple linear regression $\left(r^{2}=0.38\right)$. The slope of this line is significantly different from zero $(p<0.001)$.

Vehicles have been shown in many cases to change the skin's permeability for different substances. Hydration results from water diffusing from underlying epidermal layers or from perspiration accumulating after application of an occlusive vehicle. Under such conditions, the stratum corneum is changed from a tissue that normally contains very little water $(5-15 \%)$ to one that may contain $50 \%$ water or more. Hydration apparently 'loosens' the compact substance of the stratum corneum (Idson, 1975) and an increase in the permeability for some chemicals may thus be achieved (Behl et al., 1980; Lambert et al., 1989). In our study, changes in stripping properties occur in response to the influences of vehicles which may be interpreted as changes in the mechanical strength of the intercellular spaces and other juctional regions. An examination of Fig. 3 indicates that for treatment times of 1-4 h, stripping appears to remove more tissue in the mid-stripping region ( 5 th -7 th strip), while for treatments lasting $8-12 \mathrm{~h}$, a more uniform degree of harvest is seen over the entire 10 strips. The difference between these data sets may be due, in part, to animal and technique variability since the physical regions are proximal to each other and the total amount of tissue removed in the 10 strips from 1 to $12 \mathrm{~h}$ is relatively constant. Of far greater importance, for treatment times exceeding $12 \mathrm{~h}$, the first and second strips contained much more tissue than those that followed. An observation was also made at this point that after two strippings the skin surface appeared glistening indicating that the bulk of the stratum corneum (or epidermis) may have been stripped off (Pinkus, 1951). This seems to imply that the vehicle's effect on the skin is manifested at deeper layers of stratum corneum, possibly even to the dermo-epidermal junction, which is recognized as a weak point in the skin structure (Tregear, 1966).

We wish to emphasize that the extent to which vehicle treatment affects stripping properties may differ considerably when experiments are carried out under conditions differerent from those reported in this study. Studies on the effect of the amount of vehicle applied, composition of the vehicle, animal species used and degree of occlusion are ongoing in our laboratory. Furthermore, microscopic studies on the untreated and treated skin with successive stripping are being undertaken to further investigate the physical meaning of each tape strip. Such studies should provide a better understanding of the effect of propylene glycol/ethanol/water system on skin stripping properties.

\section{Acknowledgement}

This study was supported by The Upjohn Co., Kalamazoo, MI.

\section{References}

Behl, C.R, Flynn, G.L., Kurihara, T., Harper, N., Smith, W., Higuchi, W.I., Ho, N.F.H. and Pierson, C.L., Hydration and percutaneous absorption: I. Influence of hydration on alkanol permeation through hairless mouse skin. $J$. Invest. Dermatol., 75 (1980) 346-352.

Chiang, C.M., Flynn, G.L., Weiner, N.D. and Szpunar, G.J., Bioavailability assessment of topical delivery systems: Effect of vehicle evaporation upon in vitro delivery of minoxidil from solution formulations. Int. J. Pharm., 55 (1989) 229-236.

Han, S.R., Haberkamp, M. and Flynn G.L., Epidermal kinetics and skin condition: I. Stripping technique for quantitating 
stratum corneum turnover in hairless mouse skin. J. Toxicol-Cut. Ocular Toxicol, 8 (1989)-1990) 539-553.

Idson, B., Percutaneous absorption. J. Pharm. Sci., 64 (1975) 901-924.

Lambert, W.J., Higuchi, W.I., Knutson, K. and Krill, S.L., Effect of long-term hydration leading to the development of polar channels in hairless mouse stratum corneum. $J$. Pharm. Sci., 78 (1989) 925-928.

Pinkus, H., Examination of the epidermis by the strip method of removing horny layers $\mathrm{I}$. Observations on thickness of the horny layer, and on mitotic activity after stripping. $J$. Invest. Dermatol, 16 (1951) 383-386.

Schaefer, H., Stüttgen G., Zesch, A., Schalla, W., and Gazith J., Quantitative determination of percutaneous absorption of radiolabeled drugs in vitro and in vivo by human skin. Curr. Probl. Dermatol., 7 (1978) 80-94.

Schalla, W. and Schaefer, H., Localization of compounds in different skin layers and its use as an indicator of percuta- neous absorptions. In Bronaugh, R.L. and Maibach, H.I. (Eds), Percutaneous Absorption, Chapter 22, Dekker, New York, 1985, p. 282.

Tregear, R.T. and Dirhuber, P., The mass of keratin removed from the stratum corneum by stripping with adhesive tape. J. Invest. Dermatol., 38 (1962) 375-381.

Tregear, R.T., Physical Functions of Skin, Academic Press, New York, 1966, p. 94.

Tsai, J.C., Weiner, N.D. and Flynn, G.L., Properties of adhesive tapes used for stratum corneum stripping. Int. J. Pharm., submitted.

Weigand, D.A. and Gaylor, J.R., Removal of stratum corneum in vivo: an improvement on the cellophane tape stripping technique. J. Invest. Dermatol., 60 (1973) 84-87.

Zesch, A., Schaefer, H. and Hoffmann, W., Barrier and reservoir function of human stratum corneum for topically applied drugs. Arch. Derm. Forsch, 246 (1973) 103-107. 